\title{
Estrategias dietéticas y composición corporal en halterofilia de élite: Revisión Sistemática
}

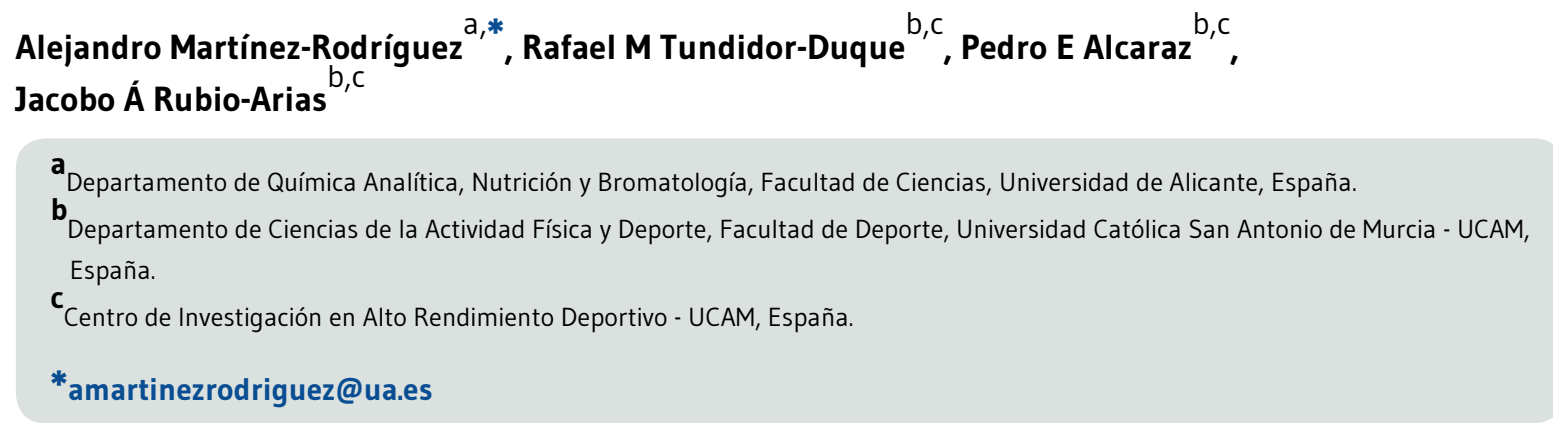

Recibido el 6 de febrero de 2017; aceptado el 22 de mayo de 2017; publicado el 29 de septiembre de 2017.

Estrategias dietéticas y composición corporal en halterofilia de élite: Revisión Sistemática

\section{PALABRAS CLAVE}

Levantamiento de Peso;

Nutrición, Alimentación y Dieta;

Tejido Adiposo;

Revisión.

\section{RESUMEN}

Introducción: Existe poca literatura que muestre las bases nutricionales para optimizar el rendimiento en halterofilia. El objetivo fue realizar una revisión sistemática de la composición corporal y composición nutricional de las dietas en halterófilos de élite.

Material y Métodos: Se revisaron artículos originales publicados en las bases de datos Pubmed, Web of Science y Sport Discuss, siguiendo los principios de la declaración PRISMA. La selección de estudios fue duplicada por dos investigadores en dos etapas (cribado y aplicación de criterios de elegibilidad). Se realizó una síntesis cualitativa de las principales características y hallazgos. Se evaluó la calidad de estudios transversales.

Resultados: De los 610 artículos encontrados, 8 cumplieron los criterios de inclusión, que fueron estudios realizados en varones halterófilos de élite 0 alto nivel y aportasen información sobre los hábitos de alimentación y/o composición corporal de los mismos. La composición corporal identificó que el porcentaje de la masa grasa promedio estuvo en torno al $15 \%$. Se registró una ingesta calórica de $4080,65 \pm 1602,69 \mathrm{kcal}$, un consumo de proteínas de $17,29 \pm 3,2 \%$ de la energía total diaria (ETD), un $42,48 \pm 5,6 \%$ ETD de hidratos de carbono y un $36,1 \pm 9,75 \%$ ETD de grasas. Los resultados sugieren que los halterófilos de élite presentan un perfil dietético subóptimo, observándose un exceso en la ingesta de grasas generalizado, lo que podría explicar el exceso de porcentaje de grasa corporal observado en algunos estudios en relación a las recomendaciones. El consumo de proteínas e hidratos de carbono estaba dentro de los rangos recomendados excepto en 2 estudios.

Conclusiones: Se observa una alimentación incorrecta por parte de los halterófilos de élite, que podría llegar a condicionar su composición corporal negativamente. Sería necesario un asesoramiento e intervención por parte de profesionales dietistas-nutricionistas. 
Dietary strategies and body composition in elite weightlifting: Systematic Review

\section{KEYWORDS}

Weight Lifting;

Athletic

Performance;

Diet, Food, and

Nutrition;

Adipose Tissue;

Review.

\section{ABSTRACT}

Introduction: There is little literature that showed the nutritional bases to optimize weightlifting performance. The objective was to perform a systematic review of the body composition and nutritional composition of diets in elite weightlifting athletes.

Material and Methods: Articles published in Pubmed, Web of Science and Sport Discuss were reviewed following PRISMA Statement. The process for selecting studies was performed duplicated by two researchers in two stages (screening and application of eligibility criteria). A qualitative synthesis of the main characteristics and findings was made. The quality of studies was assessed.

Results: Of the 610 articles found, 8 met the inclusion criteria, which were the studies performed in elite or high-level menopausal women, and information on dietary habits and/or body composition. Body composition identified that the percentage of fat mass was around $15 \%$. A caloric intake of $4080.65 \pm 1602.69 \mathrm{kcal}$, a protein intake of $17.29 \pm 3.2 \%$ of total daily energy (TDE), $42.48 \pm 5.6 \%$ TDE of carbohydrates and a $36.1 \pm 9.75 \%$ TDE of fats. A suboptimal dietary profile is suggested, showing an excess in fat intake, which could explain the excess body fat observed in some. Athletes' protein intake and carbohydrates were within the recommended ranges except for 2 studies.

Conclusions: An incorrect diet on the part of the elite weightlifting athletes was observed, which could condition their body composition negatively. The need for advice and intervention by a dietitian-nutritionist professionals was observed.

\section{CITA}

Martínez-Rodríguez A, Tundidor-Duque RM, Alcaraz PE, Rubio-Arias JA. Estrategias dietéticas y composición corporal en halterofilia de élite: Revisión Sistemática. Rev Esp Nutr Hum Diet. 2017; 21(3): 237-47. doi: 10.14306/ renhyd.21.3.353

\section{INTRODUCCIÓN}

La halterofilia es un deporte cuyo objetivo es levantar una barra con el máximo de kilos posible desde el suelo hasta por encima de la cabeza con la total extensión de los brazos $^{1}$. Existen dos levantamientos, el snatch o arrancada y el clean and jerk o dos tiempos. Tienen muchas diferencias (agarre, pico de potencia, claves en el rendimiento...) pero la principal es que en el snatch la barra se eleva directamente desde el suelo hasta encima de la cabeza con un solo movimiento mientras que en el clean and jerk son necesarios dos movimientos.

Estos movimientos han sido incluidos en muchos programas de preparación física debido a que involucran mucha masa muscular y requieren de mucha potencia para su desarrollo (resultado de la unión de cargas pesadas con mo- vimientos a altas velocidades) que se producen durante los mismos ${ }^{2}$, además existe una transferencia positiva de estos movimientos a factores de rendimiento como el salto ${ }^{3-5}$.

La halterofilia es un deporte con una gran presencia de casos de dopaje con sustancias como los esteroides anabolizantes, que podrían ayudar a la recuperación y adaptación a tales cargas de entrenamiento ${ }^{6,7}$. Estos atletas suelen permanecer en un 5-10\% por encima del peso de competición para optimizar el rendimiento en los entrenamientos ${ }^{8}$. Para dar el peso intentando mantener al máximo la masa muscular estos atletas recurren a métodos de deshidratación, como puede ser la sauna o el uso de diuréticos ${ }^{9}$. Sin embargo, se ha observado que un pequeño grado de deshidratación puede afectar al rendimiento ${ }^{10,11}$ pero con una rehidratación inmediata tras el pesaje estos efectos pueden paliarse $^{10}$ siempre y cuando el grado de deshidratación no supere el $3-4 \%$ del peso corporal. 
Estrategias dietéticas y composición corporal en halterofilia de élite: Revisión Sistemática

En esta práctica deportiva, se debería prestar especial atención a las estrategias dietético-nutricionales, que tienen como funciones fundamentales: proporcionar energía para el correcto desempeño de los entrenamientos y la competición, asegurarse una correcta recuperación y optimizar las adaptaciones producidas por el entrenamiento (como puede ser el aumento de los depósitos de fosfocreatina o la hipertrofia) $)^{12}$. Los movimientos olímpicos requieren un gran coste de energía derivada tanto del sistema de fosfágenos de alta energía ${ }^{13}$, más importante en competición debido al poco volumen de ejercicio y a los descansos completos, como de la glucolisis anaeróbica ${ }^{14,15}$ más importante a la hora de hacer frente a los grandes volúmenes de entrenamiento.

Este agotamiento de las reservas energéticas producido por el alto volumen, frecuencia e intensidad de los entrenamientos puede inducir a fatiga. Una sola sesión de entrenamiento de fuerza puede reducir las reservas de glucógeno hasta un 24-40\% ${ }^{12}$ aunque la cantidad de vaciamiento va a ser dependiente de las características del entrenamiento.

Como norma general, los halterófilos en el período preparatorio tratan de incrementar las sesiones con el objetivo instaurado en la mejora de la hipertrofia, con el fin de aumentar la masa muscular ${ }^{12}$. Por estas razones, dentro de la población de halterófilos de élite, se revisó cuáles fueron las intervenciones dietético-nutricionales o estrategias alimentarias que se llevaron a cabo sobre los mismos y qué resultados sobre la composición corporal reportaron. En este sentido, el objetivo principal de este trabajo fue realizar una revisión sistemática de la composición corporal y composición nutricional de las dietas en halterófilos de élite.

\section{MATERIAL Y MÉTODOS}

Para la realización del presente trabajo se siguieron los principios propuestos por la declaración PRISMA ${ }^{16}$. Los autores no registraron el protocolo en PROSPERO ni se publicó en ninguna revista. Las características de los estudios fueron ensayos clínicos o estudios cuasi-experimentales o transversales con deportistas de élite que practicaban halterofilia. Los criterios de inclusión fueron que los sujetos deberían ser sanos, varones y jóvenes. Los sujetos deberían estar catalogados como halterófilos de élite. Se debería de detallar al menos la composición de los macronutrientes de la dieta de los deportistas y/o composición corporal. Se excluyeron todos los estudios en los que los sujetos fueran deportistas amateur o recreacionales así como aquellos estudios en los que no apareciera ningún dato sobre aspectos nutricionales y/o composición corporal.
La búsqueda se realizó en las bases de datos Pubmed, Sport Discuss y Web of Science, con un intervalo temporal que fue del 1 enero de 1980 hasta el 31 octubre de 2016. El idioma para la selección de los artículos fue inglés o español. La identificación de estudios adicionales se formalizó mediante la herramienta de similar articles en la base de datos Pubmed. Dicha búsqueda se llevó a cabo por dos investigadores (AM y RT). Los términos que se establecieron en las estrategias de búsqueda fueron: levantamiento de peso, nutrición, dieta y composición corporal. La estrategia de búsqueda en la base de datos Pubmed fue la siguiente:

("Weight lifiting" [All Fields] OR "power lifting" [All Fields]) AND ("nutrition" [All Fields] OR "diet" [All Fields]) AND ("body composition" [All Fields])

No se incluyeron límites de diseño de estudio, edad o nivel deportivo para aumentar la sensibilidad de la búsqueda y proceder a realizar manualmente el cribado.

El proceso de selección de los estudios se realizó en dos fases, es decir el cribado (mediante la lectura del título y resumen para identificar la pertinencia del tema) y la elegibilidad de los artículos incluidos en la presente revisión sistemática. Éste se llevó a cabo por dos investigadores (AM y RT), y en caso de duda, un tercer investigador determinó la inclusión o exclusión (JAR). Se recopilaron los textos completos de los artículos preseleccionados y, posteriormente, se aplicaron los criterios de inclusión o exclusión anteriormente descritos.

Asimismo, la calidad metodológica de los estudios fue evaluada mediante la lista de 27 ítems propuesta por Berra y Cols. ${ }^{17}$ como instrumento para evaluar la calidad de estudios transversales. Este instrumento consta de 27 ítems distribuidos en 8 dimensiones denominadas: 1) pregunta u objeto de investigación; 2) participantes; 3) comparabilidad entre los grupos estudiados; 4) definición y medición de las variables principales; 5) análisis estadístico y confusión; 6) resultados; 7) conclusiones, validez externa y aplicabilidad de los resultados; 8) conflicto de intereses. Las puntuaciones posibles fueron "Muy bien", "Bien", "Regular", "Mal", "No informa" y "No aplica". La validez interna está basada en el compendio de las puntuaciones basados en los apartados originales 1-6. Mientras que la validez externa está basada en la puntuación del apartado 7. La valoración global se aplicó teniendo en cuenta todas las dimensiones del instrumento. Dicha evaluación fue realizada por dos investigadores (AM y RT), y en caso de duda, un tercer investigador determinó la clasificación (PA).

La síntesis de los resultados se realizó de forma cualitativa a través de: (a) un diagrama de flujo, donde se representó gráficamente el proceso de selección de los artículos incluidos 
en la revisión; (b) tablas de síntesis en las que se mostraron las características de los estudios incluidos en la revisión y sus correspondientes puntuaciones de la valoración crítica, y los resultados relevantes en relación a la ingesta en macro y micronutrientes de la dieta y de composición corporal.

\section{$\longrightarrow$ \\ RESULTADOS}

Como resultados, se identificaron un total de 610 artículos, de los cuales sólo se incluyeron 243 tras eliminar duplicados. En la primera fase de cribado se eliminaron 219 artículos (195 tras lectura del título y 24 tras lecturas del resumen) por no atender claramente al tema de interés. Tras la evaluación a texto completo de los 24 artículos restantes, se eliminaron 16 artículos por no cumplir con los criterios de elegibilidad 4,12,18-31, detallándose las razones de no cumplimiento en la Tabla 1. Fueron 8 los artículos finalmente incluidos en la revisión ${ }^{13,32-38}$, de los cuales se realizó un análisis y síntesis cualitativo. En la Figura 1 se detalla el proceso de selección de artículos seguido.

La Tabla 2 presenta las características de los 8 estudios incluidos en la revisión ${ }^{13,32-38}$. Se pudo observar que todos fueron estudios descriptivos y con un diseño transversal. El rango de sujetos participantes en estos estudios fue de 5-31. La edad estuvo comprendida en un rango entre 14-29 años. En ninguno de estos estudios se incluyó a mujeres como participantes en los mismos, por ello el porcentaje de estas es 0 en todos los casos. En los estudios incluidos están representados los cinco continentes (África, América, Asia, Europa y Oceanía). También se pone de manifiesto la modalidad deportiva que ocupa a esta revisión, así como el nivel de los deportistas, todos ellos halterófilos de élite. La evaluación de la dieta se realizó en la mayoría de los estudios combinando un cuestionario o entrevista semiestructurada con un registro de los alimentos consumidos por parte de los deportistas. Asimismo, en aquellos estudios que tuvieron presente la valoración de la composición corporal predominante, fue la valoración antropométrica. Sobre la calidad de las pruebas y en relación con los resultados obtenidos mediante el instrumento para la lectura crítica y la evaluación de estudios epidemiológicos transversales ${ }^{17}$. Destacar que todos los estudios obtuvieron una calificación alta o media ya que en la mayoría de los ítems obtuvieron una puntuación de "bien" (Tabla 2), a excepción de la validez interna en el caso del estudio de Burke y Cols. ${ }^{13}$.

En 6 de estos estudios se observó una distribución similar de macronutrientes (Tabla 3), con un alto consumo de grasas ( $41 \%$ del total de la energía consumida de media, llegando casi al $50 \%$ del total de calorías consumidas), mientras que solamente en el estudio de Cabral y Cols. ${ }^{32}$ y Serairi y Cols. $^{33}$ se observó que los deportistas tuvieron un consumo menor al 30\% del total calórico. Con la distribución de los

Tabla 1. Artículos eliminados y razones de exclusión.

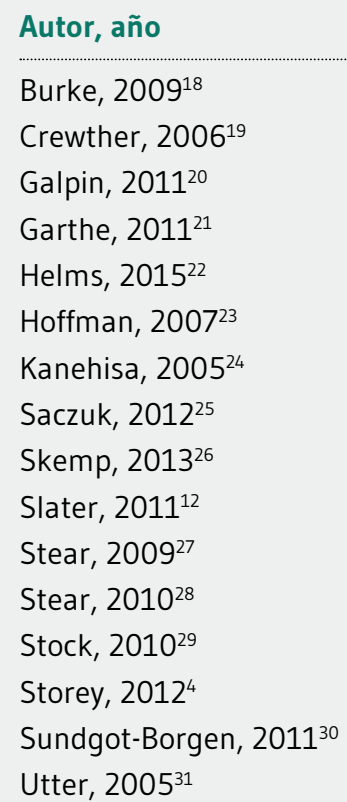

Razones exclusión

No halterofilia/rendimiento

No halterofilia

No dieta

No halterofilia/rendimiento

No halterofilia

No halterofilia/rendimiento

No dieta

No dieta

No dieta

No rendimiento

No halterofilia/rendimiento

No halterofilia/rendimiento

No halterofilia

No intervención

No rendimiento

No halterofilia 
Figura 1. Diagrama de flujo PRISMA del proceso de selección de los artículos.
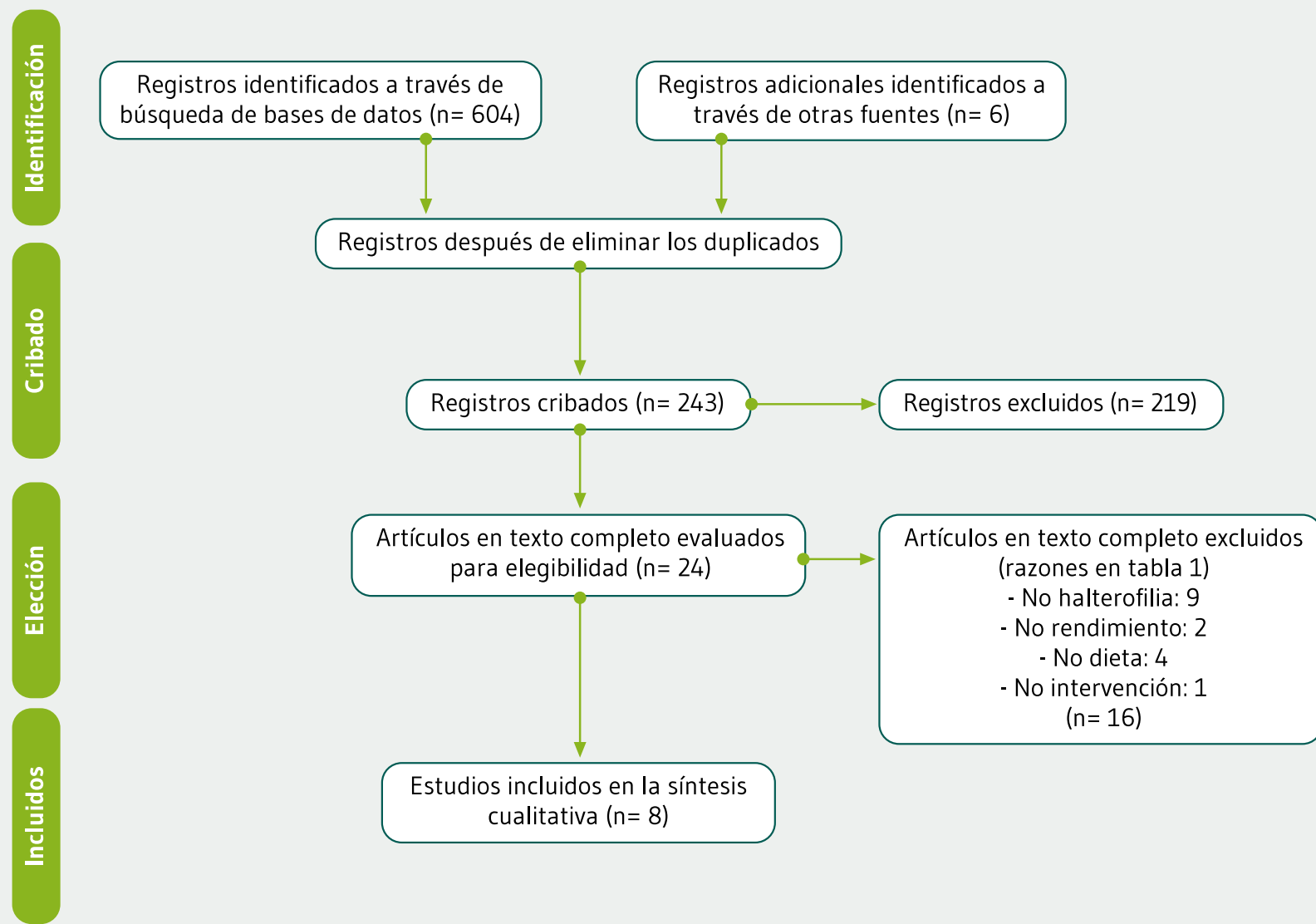

hidratos de carbono ocurrió algo similar, mostrándose en el estudio de Cabral y Cols. ${ }^{32}$, así como en el de Serairi y Cols. ${ }^{33}$ que son los únicos en los que se supera el $50 \%$ del total del aporte calórico $(5,91 \mathrm{~g} / \mathrm{kg}$ de peso y $7,5 \mathrm{~g} / \mathrm{kg}$ respectivamente), mientras que en los restantes la media fue de $40,4 \%$ del total de calorías. Sin embargo, con respecto al aporte proteico se observaron más diferencias en su consumo, con un rango que oscila entre un $12,72 \%$ hasta un $22 \%$ del total de calorías, en el que se destacan las diferencias en mayor medida cuando se calculó dicho aporte en gramos del macronutriente por kilogramos de peso (de 1,3 hasta $3,2 \mathrm{~g} / \mathrm{kg}$ de peso).

Asimismo, la Tabla 3 recopiló los datos de composición corporal de los deportistas participantes en los diferentes estudios. Los deportistas incluidos en la revisión son un total de 127. Los datos referentes a la composición corporal son diversos, encontrando un rango en el peso de 60,39 $(11,56$ hasta $118 \mathrm{~kg}$ de peso; un IMC medio de 26,32; un porcentaje de grasa que va desde el $3,6[0,8] \%$ hasta el $18,0[3,0] \%)$. Los datos referentes a la composición corporal fueron obtenidos mediante a ecuaciones estimatorias. En el trabajo de Cabral y Cols. se utilizó la ecuación basada en el trabajo de Jackson y Pollock ${ }^{39}$ para hallar la densidad corporal y posteriormente mediante una ecuación $\mathrm{n}^{40}$ estimar el porcentaje graso. Tanto en el trabajo de Hassapidou ${ }^{34}$ como en el de Serairi y Cols. ${ }^{33}$ se calculó la densidad corporal con la fórmula propuesta por Jackson y Pollock ${ }^{39}$ y posteriormente se estimó el porcentaje graso con la ecuación Siri ${ }^{41}$. En el trabajo de Burke y Cols. ${ }^{13}$ se estimó el porcentaje de grasa mediante la ecuación de Durnin y Rahaman ${ }^{42}$, basada en pliegues cutáneos al igual que la de Durnin y Womesley ${ }^{43}$ utilizada en el trabajo de van Erp-Baart ${ }^{36}$. El cálculo del porcentaje de masa libre de grasa en el trabajo de Serairi y Cols. ${ }^{33}$ fue restando el porcentaje de grasa al $100 \%$ que corresponde a todo el cuerpo. 
Rev Esp Nutr Hum Diet. 2017; 21(3): 237 - 247

Estrategias dietéticas y composición corporal en halterofilia de élite: Revisión Sistemática

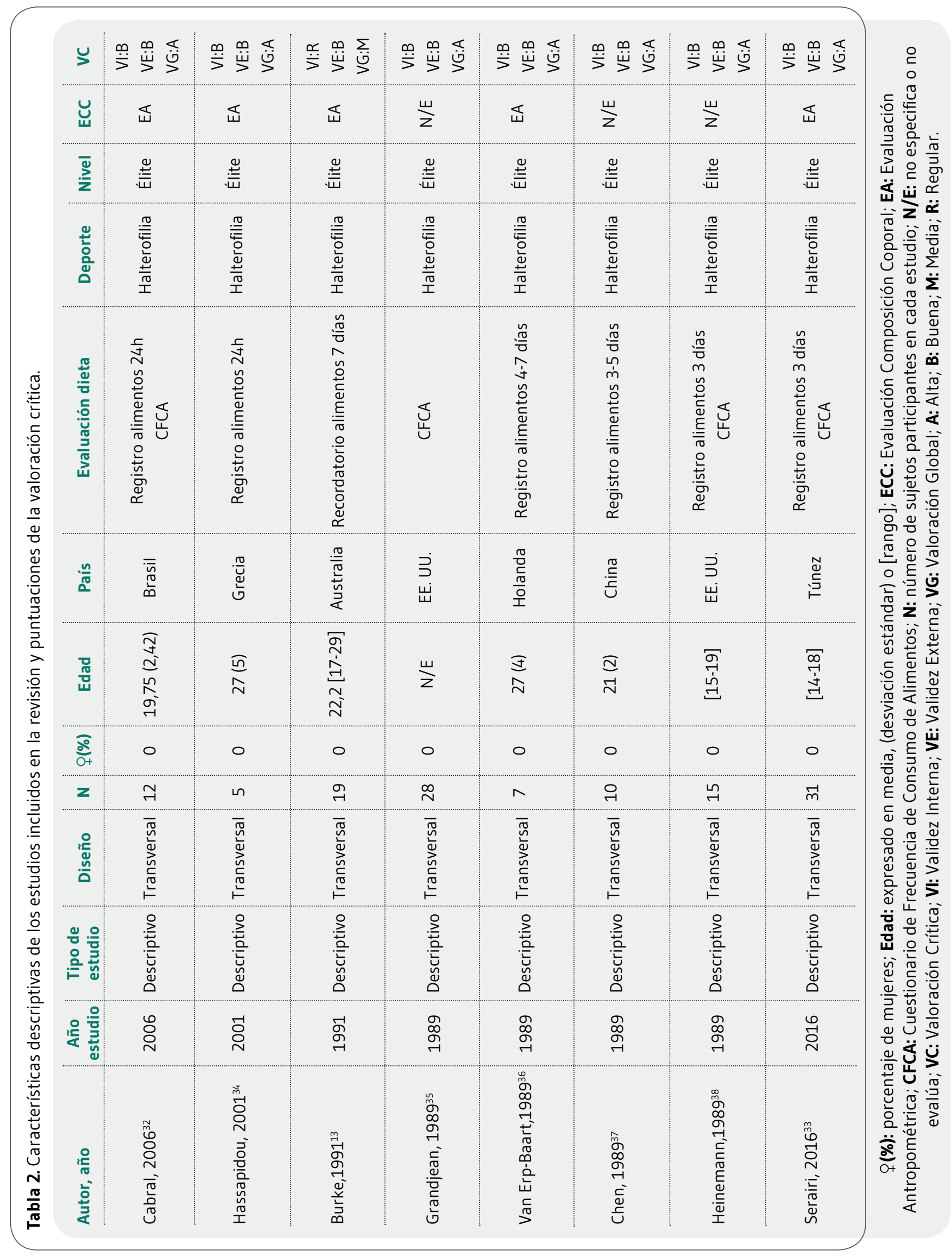


Tabla 3. Evaluación nutricional (macronoutrientes) y de la composición corporal en halterofilia de élite.

\begin{tabular}{|c|c|c|c|c|c|c|c|c|c|}
\hline & $\mathbf{N}$ & Carbohidratos & Proteínas & Lípidos & $\begin{array}{c}\text { Energía } \\
\text { (kcal) }\end{array}$ & $\begin{array}{l}\text { Altura } \\
\text { (cm) }\end{array}$ & $\begin{array}{l}\text { Peso } \\
\text { (kg) }\end{array}$ & $\begin{array}{c}\operatorname{IMC} \\
\left(\mathrm{kg} / \mathrm{m}^{2}\right)\end{array}$ & $\begin{array}{c}\text { Masa Grasa } \\
(\%)\end{array}$ \\
\hline Cabral, $2006^{32}$ & 12 & $5,9 \mathrm{~g} / \mathrm{kg}$ & $1,6 \mathrm{~g} / \mathrm{kg}$ & $28,6(5,5) \%$ & $2985(668)$ & $171,9(8,4)$ & $68,3(8,9)$ & 23,0 & $3,6(0,8)$ \\
\hline Hassapidou, $2001^{34}$ & 5 & $2,9(1,3) \mathrm{g} / \mathrm{kg}$ & $1,6(0,3) \mathrm{g} / \mathrm{kg}$ & $44(7) \%$ & $3157(420)$ & $177,0(7,0)$ & $99,0(5)$ & 31,6 & $18,0(3,0)$ \\
\hline Burke, $1991^{13}$ & 19 & $4,6 \mathrm{~g} / \mathrm{kg}$ & $1,9 \mathrm{~g} / \mathrm{kg}$ & $38,5 \%$ & 3640 & $170,2(7,9)$ & $83,8(21,1)$ & $28,6(5,1)$ & $16,5(5,8)$ \\
\hline Grandjean, $1989^{35}$ & 28 & $43,0(8,0) \%$ & $18,0(4,0) \%$ & $39(6) \%$ & $3643(927)$ & N/E & N/E & N/E & N/E \\
\hline Van Erp-Baart, $1989^{36}$ & 7 & $4,2 \mathrm{~g} / \mathrm{kg}$ & $1,3 \mathrm{~g} / \mathrm{kg}$ & $39 \%$ & 3049 & $176,4(8,4)$ & $76,4(10,0)$ & 24,6 & $15,3(3,8)$ \\
\hline Chen, $1989^{37}$ & 10 & $5,4(1,2) \mathrm{g} / \mathrm{kg}$ & $3,2(0,6) \mathrm{g} / \mathrm{kg}$ & $40(7) \%$ & $4597(604)$ & $167,0(9,9)$ & $80,0[19,0]$ & 28,7 & $N / E$ \\
\hline Heinemann, $1989^{38}$ & 15 & $8,0 \mathrm{~g} / \mathrm{kg}$ & $3,1 \mathrm{~g} / \mathrm{kg}$ & $45 \%$ & 7493 & $\mathrm{~N} / \mathrm{E}$ & $95,0[82,0-118,0]$ & N/E & N/E \\
\hline Serairi, $2016^{33}$ & 31 & $7,5 \mathrm{~g} / \mathrm{kg}$ & $2,1 \mathrm{~g} / \mathrm{kg}$ & $28(5) \%$ & 3257 & $167,0(7,0)$ & $60,4(11,6)$ & $21,5(3,4)$ & $12,3(5,2)$ \\
\hline
\end{tabular}

Valores expresados en media, (desviación estándar) o [rango]; IMC: Índice de Masa Corporal; N: Número de sujetos participantes en cada estudio; N/E: No especifica o no evalúa. Los datos de carbohidratos y proteínas en base a $\mathrm{g} / \mathrm{kg}$ de peso corporal total fueron estimados en relación al peso promedio de los deportistas.

Los datos obtenidos en relación a los micronutrientes consumidos por los deportistas participantes en cada estudio se muestran en la Tabla 4. En esta tabla se observa la distribución de las diferentes cantidades ingeridas de vitaminas y minerales. En este sentido, cabe destacar las diferencias en el consumo en vitamina $A$, tiamina, riboflavina, niacina, vitamina $C$, calcio y hierro en el estudio de Hassapidou ${ }^{34}$ frente al estudio de Burke y Cols. ${ }^{13}$, donde el consumo de estos micronutrientes es menor. Sin embargo, no se observaron tantas diferencias con respecto al estudio de Chen y Cols. ${ }^{37}$. En relación a los datos comunes a todos los estudios incluidos, incidir en el calcio, donde aparentemente

Tabla 4. Evaluación nutricional (micronutrientes) en halterofilia de élite.

\begin{tabular}{|c|c|c|c|c|}
\hline & Hassapidou, $2001^{34}$ & Burke, $1991^{13}$ & Chen, $1989^{37}$ & Serairi, $2016^{33}$ \\
\hline N & 5 & 19 & 10 & 31 \\
\hline Vitamina A & $5,9(1,6)$ чg & $132,0(68,0)$ чg & $1547,0(99,0)(\mathrm{mg})$ & N/E \\
\hline Tiamina (mg) & $2,5(0,5)$ & $0,1(0,1)$ & $1,8(0,3)$ & N/E \\
\hline Riboflavina (mg) & $2,7(0,8)$ & $0,2(0,1)$ & $2,7(0,6)$ & N/E \\
\hline Niacina (mg) & $33,0(5,0)$ & $3,7(1,4)$ & $36,3(8,0)$ & N/E \\
\hline Vitamina C (mg) & $168,0(81,0)$ & $13,0(8,0)$ & $93,0(35,0)$ & N/E \\
\hline Vitamina E (mg) & $15,0(4,0)$ & N/E & N/E & N/E \\
\hline Vitamina B6 (mg) & $2,0(0,9)$ & N/E & N/E & N/E \\
\hline Vitamina B12 (4g) & $8,5(3,0)$ & N/E & N/E & N/E \\
\hline Calcio (mg) & $1,6(181,0)$ & $103,0(55,0)$ & $1597,0(195,0)$ & $822,51(188,6)$ \\
\hline Zinc (mg) & $23(7,0)$ & $N / E$ & N/E & N/E \\
\hline Hierro (mg) & $22(3,0)$ & $1,5(0,4)$ & 50,0 (9) & 20,5 \\
\hline Potasio (g) & $N / E$ & N/E & $7,5(2,4)$ & $3718(1075,2)$ \\
\hline Sodio (g) & N/E & N/E & $7,0(0,6)$ & \\
\hline Magnesio (mg) & N/E & N/E & $777,0(304,0)$ & $199,9(42,6)$ \\
\hline
\end{tabular}

Valores expresados en media (desviación estándar); N: número de sujetos participantes en cada estudio;

N/E: no especifica o no evalúa. 
Ios halterófilos participantes en el estudio de Chen y Cols. ${ }^{37}$ consumieron una mayor cantidad de calcio en comparación con el resto, siendo los halterófilos participantes en el estudio de Hassapidou ${ }^{34}$ los que menos calcio consumieron. No sucedió de igual forma en relación con el hierro, donde los halterófilos del estudio de Burke y Cols. ${ }^{13}$ mostraron cantidades inferiores al resto, y nuevamente los halterófilos del estudio de Chen y Cols. ${ }^{37}$ fueron los que más cantidad de hierro consumieron.

\section{$\longrightarrow$ DISCUSIÓN}

Los resultados obtenidos plantearon, en líneas generales, por un lado, que la composición nutricional de la dieta de los halterófilos está basada en una ingesta energética elevada, por encima de las $3.000 \mathrm{kcal}$ en todos los casos, con una distribución de macronutrientes en las que predomina el aporte de carbohidratos (mayor del 50\% kcal totales), seguidos de grasas ( $\geq 30 \%$ ) y proteínas (aproximadamente $1,6-3 \mathrm{~g} / \mathrm{kg}$ de peso). Y por otro, que en la composición corporal, los halterófilos, presentaron resultados con una proporción de masa grasa considerable, por encima del $10 \%$ pero por debajo del $20 \%$.

Según la bibliografía específica en el tema de la presente revisión sistemática, los requerimientos energéticos de la población de halterófilos de élite abogan por una ingesta de hidratos de carbono de entre $4-7 \mathrm{~g} / \mathrm{kg}$ de peso, periodizando la ingesta con respecto al gasto energético del deportista en cada momento de la temporada. Como se ha observado en el presente estudio, estos deportistas han estado siempre asociados a grandes ingestas de proteínas, siendo las recomendaciones actuales para deportistas de modalidades de fuerza $1,6-2 \mathrm{~g} / \mathrm{kg}$ de peso ${ }^{12,44}$. Consumos por encima de este rango parecen no ofrecer ventajas, y aumentan el catabolismo proteico ${ }^{45}$. Además, en relación con el desarrollo muscular y rendimiento deportivo, parece ser que cuanto más avanza la temporada y el atleta se va a adaptando al entrenamiento, las necesidades proteicas se ven ligeramente reducidas ${ }^{46}$. En este sentido, cabe destacar no sólo la cantidad total de nutriente que es ingerido, sino el momento en el que se hace ${ }^{47}$. Por ejemplo, en cuanto a unas posibles recomendaciones para halterófilos de élite relacionadas con el momento de la ingesta, incidir en que la comida post-entrenamiento debe tener el objetivo de recuperar los depósitos de energía y favorecer las adaptaciones del entrenamiento ${ }^{47,48}$. En este sentido, la ingesta de hidratos de carbono junto a proteínas parece la estrategia más recomendable. Una combinación de $0,8-1,2 \mathrm{~g} / \mathrm{kg}$ de peso de hidratos de carbono más $0,4 \mathrm{~g} / \mathrm{kg}$ de peso de proteína de alta calidad parece ser una buena estrategia para reponer los depósitos de glucógeno, así como para estimular la síntesis proteica ${ }^{12}$. El resto de la ingesta de nutrientes debe de ser correspondido a las grasas, con una ingesta de entre el $20-30 \%$ del total calórico ${ }^{44}$, debido a sus importantes funciones sobre el organismo, como el transporte de vitaminas liposolubles o la formación de membranas celulares ${ }^{12,44}$. En los estudios incluidos en la revisión, se observaron ingestas de grasas por encima de los valores recomendados en más de un grupo de deportistas, lo que podría explicar el incremento del porcentaje de masa grasa por encima de los valores de referencia hallados en algunos estudios.

Esta revisión parece indicar, en general, un mal perfil de la dieta o perfil subóptimo por parte de los halterófilos de élite, sin embargo se observó una tendencia en los estudios más actuales $^{32,33}$ de unos perfiles de la dieta más acordes con la actividad de estos deportistas. Solamente en el trabajo de Cabral y Cols. $^{32}$ así como en el de Serairi y Cols. ${ }^{33}$ la distribución de macronutrientes es afín a lo recomendado por la literatura científica ${ }^{12}$. Se observó un consumo de $1,59 \mathrm{~g} / \mathrm{kg}$ de proteínas, $5,91 \mathrm{~g} / \mathrm{kg}$ de hidratos de carbono y $28,57 \%$ de la ingesta calórica dedicada a las grasas ${ }^{32}$ y un consumo de $2,05 \mathrm{~g} / \mathrm{kg}$ de proteínas, $7,5 \mathrm{~g} / \mathrm{kg}$ de hidratos de carbono y un $28,53 \%$ de consumo de grasas respecto al total ${ }^{33}$. Pero al estudiar las necesidades calóricas diarias de los sujetos, los investigadores observaron que tenían un déficit energético, calculado en función a la ecuación de la $\mathrm{OMS}^{49}$ y con el factor de actividad propuesto por James y Schofield ${ }^{50}$ de 679 calorías, lo que podría explicar los porcentajes de grasa tan

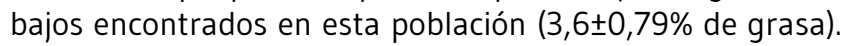
Mientas que en el resto de estudios ${ }^{13,34,39}$ la ingesta calórica parece superior a las necesidades de estos deportistas y/o la distribución de macronutrientes no es óptima debido a que los porcentajes de grasa corporal son elevados. El porcentaje de grasa ideal para deportes de fuerza en los que existen categorías de peso parece ser un $10-12 \%$ de grasa corporal $^{32}$ para optimizar la relación peso corporal/fuerza que es clave en el rendimiento ${ }^{13}$, excepto en la categoría sin límite de peso (más de 105 kilogramos). En el trabajo de Serairi y Cols. ${ }^{33}$ sí se encontraron porcentajes de grasa corporal aceptables en función de lo que dice la literatura científica. Esto se puede explicar debido al correcto reparto de macronutrientes en la dieta de estos deportistas.

Sólo se presentaron dos estudios fuera de los límites del consumo de hidratos de carbono recomendado $\left(2,9 \pm 1,3 \mathrm{~g} / \mathrm{kg}^{34}\right.$ y $\left.8 \mathrm{~g} / \mathrm{kg}^{38}\right)$. Tanto un consumo por encima como por debajo puede afectar al rendimiento y/o salud del deportista, afectando a la correcta recuperación y reposición de los sustratos energéticos (consumo bajo) o pudiendo producir un aumento no deseado de peso (consumo excesivo). En 3 de los estudios ${ }^{13,35,36}$ que están dentro del rango recomendado 
(4-7g/kg de peso) el consumo está muy cerca del límite inferior a pesar de que los porcentajes de grasa corporal son altos. Esto puede deberse al alto consumo de grasas. Sería recomendable bajar el consumo de grasas en favor del consumo de hidratos de carbono, debido al carácter anaeróbico de este deporte y a los entrenamientos caracterizados por alta frecuencia, alto volumen y alta intensidad.

En cuanto al consumo de proteínas, existen estudios ${ }^{13,33,34,36}$ que cumplen los estándares anteriormente expuestos del rango $1,6-2 \mathrm{~g} / \mathrm{kg}$ de peso ${ }^{12,44}$, pero también existen 2 estu$\operatorname{dios}^{37,38}$ que muestran un excesivo consumo de este macronutriente (con más de $3 \mathrm{~g} / \mathrm{kg}$ de peso); lo cual no ofrece ninguna ventaja sobre consumos más moderados de proteínas ${ }^{45}$, pero tampoco parece ser un riesgo para la salud del deportista ${ }^{51}$. Aun así sería interesante disminuir el consumo de proteínas en estos sujetos bajando el consumo de productos animales, lo que podría ser la razón del alto consumo de grasas y porque este consumo excesivo promueve un catabolismo proteico excesivo ${ }^{45}$.

El consumo de grasas es superior a las recomendaciones del $30 \%$ del total calórico 45,50 menos en dos $\operatorname{casos}^{32,33}$. En todos los demás estudios se muestran ingestas por encima del $35 \%$ del total calórico, lo cual podría afectar al rendimiento ya que puede ser la razón de los elevados porcentajes de grasa corporal, y tales consumos de grasas no tiene ningún efecto positivo sobre el rendimiento deportivo ya que en la halterofilia es un deporte puramente anaeróbico.

Con respecto al aporte de vitaminas y minerales, sería interesante haber podido conocer el aporte de micronutrientes en todos los estudios, lo cual delimita y dificulta la comparación de los mismos con valores de referencias o entre diferentes poblaciones de deportistas de élite que realicen halterofilia. No obstante, destacar que entre las limitaciones del presente trabajo se encontraron el reducido número de artículos que tratan específicamente la composición nutricional de las dietas y la composición corporal de los halterófilos de élite. Asimismo, aparecen problemas en relación a la correcta cuantificación de los macronutrientes y micronutrientes ingeridos por los deportistas, debido a la heterogeneidad de los métodos para conocer dichas cantidades. Del mismo modo, en relación a la composición corporal, no se detallan en los artículos todos los datos de cómo se han llevado a cabo las valoraciones antropométricas y los resultados obtenidos a partir de estos.

Como futuras investigaciones, se podría plantear que se pusieran de manifiesto distintas distribuciones de macronutrientes y/o distintos balances energéticos y su influencia sobre el rendimiento (en este caso a los kg levantados en los movimientos olímpicos). Asimismo, se podría estudiar la influencia de distintas comidas pre- y post-entrenamiento en el rendimiento y fatiga de los atletas, observando los distintos marcadores biológicos (testosterona, cortisol, otros) para poder estudiar también el estado de salud del deportista y la respuesta a tales niveles de entrenamiento con unas y otras comidas. Además de la influencia o no en el rendimiento del uso de distintos suplementos que teóricamente deberían aumentar el rendimiento. Sería interesante que pudiesen abordar con más precisión el tema en cuestión, con la intención de poder facilitar a los dietistas-nutricionistas unas recomendaciones más exhaustivas sobre los requerimientos en esta población de deportistas. No obstante, en líneas generales, en este manuscrito se han recopilado algunas de estas estrategias y que pueden ser de ayuda para el colectivo profesional de dietistas-nutricionistas.

\section{CONCLUSIONES}

A pesar de que en general la ingesta proteica de los halterófilos de élite estuvo dentro de los rangos recomendados, en 2 estudios se reportaron ingestas de hasta $1 \mathrm{~g} / \mathrm{kg}$ peso superiores a las recomendaciones. De forma generalizada, el dato más alterado en las dietas de los deportistas, fue el elevado consumo de grasa, situado alrededor de un $40 \%$ en la mayoría de los casos. Este exceso en la ingesta de lípidos también contribuye al detrimento del aporte de hidratos de carbono en esta población. En futuros trabajos se debería estudiar si este hecho podría relacionarse con el contenido en masa grasa, ya que el compartimento de masa grasa se sitúa en un rango entre el $10-20 \%$ del peso corporal total. En cuanto al aporte en micronutrientes, es necesario llevar a cabo más investigaciones centradas en este aspecto. Es necesario recomendar el papel del dietista-nutricionista en la preparación de los halterófilos de élite para llevar a cabo una adecuada planificación dietético-nutricional acorde con el requerimiento de la práctica deportiva, que ayude a estos deportistas a maximizar su rendimiento deportivo, tanto en los entrenamientos como durante la competición, incrementando la masa muscular y disminuyendo la masa grasa.

\section{$\longrightarrow$ CONFLICTO DE INTERESES}

Los autores expresan que no hay conflictos de interés al redactar el manuscrito. 


\section{REFERENCIAS}

((1) Everett G. Olympic Weightlifting: a Complete Guide for Athletes \& Coaches. $2^{a}$ ed. Sunnyvale, Calif.: Catalyst Athletics; 2012.

(2) Garhammer J. Power production by Olympic weightlifters. Med Sci Sports Exerc. 1980; 12(1): 54-60.

(3) Baker D, Nance S. The Relation Between Strength and Power in Professional Rugby League Players. J Strength Cond Res. 1999; 13(3): 224.

(4) Storey A, Smith HK. Unique aspects of competitive weightlifting: performance, training and physiology. Sports Med. 2012; 42(9): 769-90.

(5) Hamill BP. Relative Safety of Weightlifting and Weight Training. J Strength Cond Res. 1994; 8(1): 53-7.

(6) Stone MH, Pierce KC, Sands WA, Stone ME. Weightlifting: A Brief Overview. Strength Cond J. 2006; 28(1): 50-66.

(7) Franke WW, Berendonk B. Hormonal doping and androgenization of athletes: a secret program of the German Democratic Republic government. Clin Chem. 1997; 43(7): 1262-79.

(8) Rogozkin VA. Weightlifting and Power Events. En: J.ughan R, editor. Nutrition in Sport [Internet]. Malden, MA: Blackwell Science Ltd; 2000. p. 622-31. Disponible en: http:// onlinelibrary.wiley.com/doi/10.1002/9780470693766.ch47/ summary

(9) Judelson DA, Maresh CM, Anderson JM, Armstrong LE, Casa D], Kraemer W], et al. Hydration and muscular performance: does fluid balance affect strength, power and high-intensity endurance? Sports Med. 2007; 37(10): 907-21.

(10) Schoffstall JE, Branch JD, Leutholtz BC, Swain DE. Effects of dehydration and rehydration on the one-repetition maximum bench press of weight-trained males. ] Strength Cond Res. 2001; 15(1): 102-8.

(11) Judelson DA, Maresh CM, Farrell MJ, Yamamoto LM, Armstrong $L E$, Kraemer W], et al. Effect of hydration state on strength, power, and resistance exercise performance. Med Sci Sports Exerc. 2007; 39(10): 1817-24.

(12) Slater G, Phillips SM. Nutrition guidelines for strength sports: sprinting, weightlifting, throwing events, and bodybuilding. J Sports Sci. 2011; 29 Suppl 1: S67-77.

(13) Burke LM, Gollan RA, Read RS. Dietary intakes and food use of groups of elite Australian male athletes. Int J Sport Nutr. 1991; 1(4): 378-94.

(14) Lambert CP, Flynn MG. Fatigue during high-intensity intermittent exercise: application to bodybuilding. Sports Med. 2002; 32(8): 511-22.

(15) Tesch PA, Colliander EB, Kaiser P. Muscle metabolism during intense, heavy-resistance exercise. Eur ] Appl Physiol Occup Physiol. 1986; 55(4): 362-6.

(16) Urrútia G, Bonfill X. Declaración PRISMA: una propuesta para mejorar la publicación de revisiones sistemáticas y metaanálisis. Med Clin (Barc). 2010; 135(11): 507-11.

(17) Berra S, Elorza-Ricart JM, Estrada M-D, Sánchez E. Instrumento para la lectura crítica y la evaluación de estudios epidemiológicos transversales. Gac Sanit. 2008; 22(5): 492-7.

(18) Burke LM, Castell LM, Stear SJ. BJSM reviews: A-Z of supplements: dietary supplements, sports nutrition foods and ergogenic aids for health and performance Part 1. Br J Sports Med. 2009; 43(10): 728-9.

(19) Crewther B, Keogh J, Cronin J, Cook C. Possible stimuli for strength and power adaptation: acute hormonal responses. Sports Med. 2006; 36(3): 215-38.

(20) Galpin AJ, Fry AC, Chiu LZF, Thomason DB, Schilling BK. Highpower resistance exercise induces MAPK phosphorylation in weightlifting trained men. Appl Physiol Nutr Metab. 2012; 37(1): 80-7.

(21) Garthe I, Raastad T, Sundgot-Borgen J. Long-term effect of weight loss on body composition and performance in elite athletes. Int J Sport Nutr Exerc Metab. 2011; 21(5): 426-35.

(22) Helms ER, Zinn C, Rowlands DS, Naidoo R, Cronin J. Highprotein, low-fat, short-term diet results in less stress and fatigue than moderate-protein moderate-fat diet during weight loss in male weightlifters: a pilot study. Int ] Sport Nutr Exerc Metab. 2015; 25(2): 163-70.

(23) Hoffman JR. Protein Intake: Effect of Timing. Strength Cond J. 2007; 29(6): 26.

(24) Kanehisa H, Funato K, Abe T, Fukunaga T. Profiles of muscularity in junior Olympic weight lifters. J Sports Med Phys Fitness. 2005; 45(1): 77-83.

(25) Saczuk J, Wasiluk A. Dependence between Body Tissue Composition and Results Achieved by Weightlifters. Balt ] Health Phys Act. 2012; 4(1): 15-20.

(26) Skemp KM, Mikat RP, Schenck KP, Kramer NA. Muscle dysmorphia: risk may be influenced by goals of the weightlifter. J Strength Cond Res. 2013; 27(9): 2427-32.

(27) Stear SJ, Burke LM, Castell LM. BJSM reviews: A-Z of nutritional supplements: dietary supplements, sports nutrition foods and Ergogenic aids for health and performance Part 3. Br J Sports Med. 2009; 43(12): 890-2.

(28) Stear SJ, Castell LM, Burke LM, Spriet LL. BJSM reviews: A-Z of nutritional supplements: dietary supplements, sports nutrition foods and ergogenic aids for health and performance Part 6. Br J Sports Med. 2010; 44(4): 297-8.

(29) Stock MS, Young JC, Golding LA, Kruskall L], Tandy RD, Conway-Klaassen JM, et al. The effects of adding leucine to pre and postexercise carbohydrate beverages on acute muscle recovery from resistance training. ] Strength Cond Res. 2010; 24(8): 2211-9.

(30) Sundgot-Borgen J, Garthe I. Elite athletes in aesthetic and Olympic weight-class sports and the challenge of body weight and body compositions. J Sports Sci. 2011; 29 Suppl 1: S101-114.

(31) Utter AC, Kang J, Nieman DC, Brown VA, Dumke CL, McAnulty $\mathrm{SR}$, et al. Carbohydrate supplementation and perceived exertion during resistance exercise. J Strength Cond Res. 2005; 19(4): 939-43.

(32) Cabral CAC, Rosado GP, Silva CHO, Marins JCB. Diagnosis of the nutritional status of the Weight Lifting Permanent Olympic Team athletes of the Brazilian Olympic Committee (COB). Rev Bras Med Esporte. 2006; 12(6):3 45-50.

(33) Serairi Beji R, Megdiche Ksouri W, Ben Ali R, Saidi O, Ksouri $\mathrm{R}$, Jameleddine $\mathrm{S}$. Evaluation of nutritional status and body composition of young Tunisian weightlifters. Tunis Med. 2016; 94(2): 112-7.

(34) Hassapidou M. Dietary assessment of five male sports teams in Greece. Nutr Food Sci. 2001; 31(1): 31-5.

(35) Grandjean AC. Macronutrient intake of US athletes compared 
with the general population and recommendations made for athletes. Am J Clin Nutr. 1989; 49(5 Suppl): 1070-6.

(36) van Erp-Baart AM, Saris WH, Binkhorst RA, Vos JA, Elvers JW. Nationwide survey on nutritional habits in elite athletes. Part I. Energy, carbohydrate, protein, and fat intake. Int J Sports Med. 1989; 10(Suppl 1): S3-10.

(37) Chen JD, Wang JF, Li KJ, Zhao YW, Wang SW, Jiao Y, et al. Nutritional problems and measures in elite and amateur athletes. Am J Clin Nutr. 1989; 49(5 Suppl): 1084-9.

(38) Heinemann L, Zerbes H. Physical activity, fitness, and diet: behavior in the population compared with elite athletes in the GDR. Am J Clin Nutr. 1989; 49(5 Suppl): 1007-16.

(39) Jackson AS, Pollock ML. Generalized equations for predicting body density of men. $\mathrm{Br}]$ Nutr. 1978; 40(3): 497-504.

(40) Heyward VH, Stolarczyk LM. Avaliaçao da composiçâo corporal aplicada. São Paulo: Manole; 2000.

(41) McArdle WD, Katch Fl, Katch VL. Essentials of exercise physiology. 3a ed. Philadelphia, Pa.: Lippincott Williams \& Wilkins; 2006.

(42) Durnin JV, Rahaman MM. The assessment of the amount of fat in the human body from measurements of skinfold thickness. Br J Nutr. 1967; 21(3): 681-9.

(43) Durnin JV, Womersley J. Body fat assessed from total body density and its estimation from skinfold thickness: measurements on 481 men and women aged from 16 to 72 years. Br J Nutr. 1974; 32(1): 77-97.

(44) Thomas DT, Erdman KA, Burke LM. American College of Sports Medicine Joint Position Statement. Nutrition and Athletic Performance. Med Sci Sports Exerc. 2016; 48(3): 543-68.
(45) Moore DR, Robinson MJ, Fry JL, Tang JE, Glover El, Wilkinson $\mathrm{SB}$, et al. Ingested protein dose response of muscle and albumin protein synthesis after resistance exercise in young men. Am J Clin Nutr. 2009; 89(1): 161-8.

(46) Hartman JW, Moore DR, Phillips SM. Resistance training reduces whole-body protein turnover and improves net protein retention in untrained young males. Appl Physiol Nutr Metab. 2006; 31(5): 557-64.

(47) Aragon AA, Schoenfeld BJ. Nutrient timing revisited: is there a post-exercise anabolic window? J Int Soc Sports Nutr. 2013; 10(1): 5 .

(48) Ivy JL, Ding Z, Hwang H, Cialdella-Kam LC, Morrison PJ. Post exercise carbohydrate-protein supplementation: phosphorylation of muscle proteins involved in glycogen synthesis and protein translation. Amino Acids. 2008; 35(1): 89-97.

(49) World Health Organization. Energy and protein requirements. Report of a joint FAO/WHO/UNU Expert Consultation. World Health Organ Tech Rep Ser. 1985; 724.

(50) James WPT, Schofield C, Food and Agriculture Organization of the United Nations. Human energy requirements: a manual for planners and nutritionists. Oxford: FAO and Oxford University Press; 1990.

(51) Antonio J, Peacock CA, Ellerbroek A, Fromhoff B, Silver T. The effects of consuming a high protein diet $(4.4 \mathrm{~g} / \mathrm{kg} / \mathrm{d})$ on body composition in resistance-trained individuals. J Int Soc Sports Nutr. 2014; 11: 19. 